\title{
Light-assisted drying for protein stabilization
}

Madison A. Young Andrew T. Antczak

Amanda Wawak

Gloria D. Elliott

Susan R. Trammell 


\title{
Light-assisted drying for protein stabilization
}

\author{
Madison A. Young, ${ }^{a}$ Andrew T. Antczak, ${ }^{a}$ Amanda Wawak, ${ }^{a}$ Gloria D. Elliott, ${ }^{b}$ and Susan R. Trammell ${ }^{a, *}$ \\ aUniversity of North Carolina at Charlotte, Department of Optical Science and Engineering, Charlotte, North Carolina, United States \\ bUniversity of North Carolina at Charlotte, Department of Mechanical Engineering, Charlotte, North Carolina, United States
}

\begin{abstract}
A light-based processing method to create an amorphous trehalose matrix for the stabilization of proteins is discussed. This method has potential applications in the stabilization of protein-based therapeutics and diagnostics. During light-assisted drying (LAD), proteins suspended in a trehalose solution are dehydrated using near-infrared (NIR) laser light. The goal of this study was to determine processing parameters that resulted in fast processing times and low end moisture contents (EMC), while maintaining the functionality of embedded proteins. We compared the effect of changing processing wavelength, power and resulting sample temperature, and substrate material on the EMC for two NIR laser sources (1064 and $1850 \mathrm{~nm}$ ). The 1850-nm laser resulted in the lowest EMC $\left(0.03 \pm 0.01 \mathrm{gH}_{2} \mathrm{O} / \mathrm{gDryWeight}\right)$ after $20 \mathrm{~min}$ of processing on glass microfiber paper. This suggests a storage temperature of $68.3^{\circ} \mathrm{C}$. We also tested the functionality of a model protein, lysozyme, after LAD processing using a standard assay. LAD showed no significant effect on the functionality of lysozyme when processed at a maximum temperature of $\sim 44^{\circ} \mathrm{C}$ to an EMC of $0.17 \pm 0.06 \mathrm{gH}_{2} \mathrm{O} / \mathrm{gDryWeight}$. LAD is a promising technique for forming amorphous trehalose solids that could stabilize proteins at ambient temperatures. (C) 2018 Society of Photo-Optical Instrumentation Engineers (SPIE) [DOI: 10.1117/1.JBO.23.7.075007]
\end{abstract}

Keywords: amorphous solids; near-infrared irradiation; anhydrous preservation; selective heating; protein stabilization.

Paper 180260R received May 2, 2018; accepted for publication Jun. 28, 2018; published online Jul. $18,2018$.

\section{Introduction}

The use of protein-based therapeutics has increased dramatically since the introduction of the first recombinant protein therapeutic-human insulin-more than 30 years ago. ${ }^{1}$ Since then, protein therapeutics have been developed to treat diseases ranging from arthritis and psoriasis to cancer. ${ }^{2}$ Proteins are also used in diagnostics, such as microarrays, which contain immobilized capture proteins for the detection of diseases. ${ }^{3}$ A challenge in the development of protein-based diagnostics and drugs is maintaining the protein in the folded state during processing and storage as the three-dimensional structure of the protein is often responsible for its functional activity.,

Long shelf-lives for proteins have been achieved by freeze drying, also known as lyophilization. This is currently the gold standard for preparing proteins for long-term storage; however, the complexity, processing time, high cost, and potential for instability during processing and storage are disadvantages of this technique. ${ }^{6-8}$ Further, even after lyophilization, many proteins still have to be stored below $4^{\circ} \mathrm{C} .{ }^{9}$ Protein therapeutics and diagnostics would benefit from a quick, relatively inexpensive processing method that could also enable higher temperature storage.

Recent research has demonstrated that anhydrous, or dry state, preservation in a trehalose amorphous solid matrix may be an alternative to freeze drying for the preservation of biological samples. ${ }^{10}$ An amorphous solid restricts molecular motion to a small volume over a finite time period, which can prevent the degradation of biologics, such as proteins, embedded in the matrix. An amorphous solid is a noncrystalline solid, in which the atoms and molecules are organized such that there is no

*Address all correspondence to: Susan R. Trammell, E-mail: srtramme@uncc .edu long-range order. ${ }^{11}$ The regular lattice of a crystalline solid can damage embedded biologics, limiting the usefulness of these solids as preservation matrices. Disaccharide trehalose can form a low mobility glass (amorphous solid) at room temperature and can also act as a bioprotectant, making trehalose an attractive option as a preservation matrix for embedded biologics. ${ }^{12}$ Trehalose is thought to protect biologics during dehydration by compensating for the loss of hydrogen bonding with water on the surface of folded proteins without changing their conformation. ${ }^{13}$ Removing water from a trehalose solution forms an amorphous solid preservation matrix. As water is removed from the sample, the remaining sugars and salts become concentrated, and, as long as the solutes do not crystallize, the viscosity increases with progressive water loss until an amorphous solid is achieved.

Because a substantial reduction of molecular mobility is necessary to ensure an extended shelf life, samples generally need to be stored below the glass transition temperature $\left(T_{\mathrm{g}}\right)$ of the trehalose matrix to prevent degradation. ${ }^{14}$ Below $T_{\mathrm{g}}$, the trehalose maintains its amorphous state. The Gordon-Taylor ${ }^{15}$ equation can be used to predict the glass transition temperature $\left(T_{\mathrm{g}}\right)$ of trehalose-water mixturesr15

$T_{\mathrm{g}}=\frac{x_{1} T_{\mathrm{g}, 1}+k_{\mathrm{GT}}\left(1-x_{1}\right) T_{\mathrm{g}, 2}}{x_{1}+k_{\mathrm{GT}}\left(1-x_{1}\right)}$.

The glass transition temperatures of pure trehalose and pure water are given by $T_{\mathrm{g}, 1}$ and $T_{\mathrm{g}, 2}$, respectively, $x_{1}$ is the weight fraction of trehalose, and $k_{\mathrm{GT}}$ is an empirically determined fitting parameter of 5.2. ${ }^{16}$ The glass transition temperature for an amorphous trehalose solid formed by dehydration depends on

1083-3668/2018/\$25.00 @ 2018 SPIE 
the amount of water remaining in the sample after processing. The more water that remains in the sample, the lower the glass transition temperature. Lower moisture contents are necessary for storage at higher temperatures. Achieving these low end moisture contents (EMC), while maintaining protein functionality, is the key to success for anhydrous preservation methods.

Other researchers have devised ways to form trehalose amorphous solids for use as preservation matrices. These include drying in controlled humidity desiccators ${ }^{17}$ and the use of high flow nitrogen gas for convective drying. ${ }^{18}$ Recently, Cellemme et al. ${ }^{19}$ have had success with microwave-assisted drying of small volume biologics. All of these techniques are limited to batch processing. Light-assisted drying (LAD) could be used for inline processing and has the benefit of being able to deliver more precise amounts of energy to each sample. Precise control over energy deposition means precise control over EMC, which has implications on sample storage temperature.

In this study, we use a technique, LAD, to form an amorphous trehalose solid. LAD uses illumination by near-infrared laser light to assist in the formation of trehalose amorphous solids. Static air drying of sugar solutions is dominated by evaporative cooling, which causes the drying rate to slow substantially and allows for crystallization of the sugars. LAD selectively heats water to overcome cooling due to evaporation and speeds dehydration of the samples. Two laser sources at 1064- and 1850 -nm wavelengths were used to heat water in small volume samples. These two wavelengths have different absorption coefficients in water $\left(0.12\right.$ and $1.2 \mathrm{~cm}^{-1}$, respectively) and were chosen to test the effect of optical penetration depth in a sample on the resultant EMC. There is little absorption of key subcellular components, such as DNA and proteins, at these wavelengths. ${ }^{20}$ In this paper, the water content of trehalose glasses formed via LAD using different laser wavelengths (1064 and $1850 \mathrm{~nm}$ ), laser processing powers, and processing times as well as their thermal histories during processing are presented. In addition, two drying substrates-borosilicate glass coverslips and a microfiber filter paper-were tested. Predicted glass transition temperatures for the trehalose preservation matrices produced via LAD are calculated and discussed. We also present initial functionality tests of the protein lysozyme after LAD processing.

\section{Methods}

\subsection{Light-Assisted Drying Processing}

A schematic of the experimental setup is shown in Fig. 1. Two IPG Photonics laser sources were used separately for LAD processing: a continuous wave $(\mathrm{CW})$ ytterbium fiber laser at $1064 \mathrm{~nm}$ (YLR-5-1064) and a CW thulium fiber laser at $1850 \mathrm{~nm}$ (TLM-5). Both sources have maximum power outputs of $5 \mathrm{~W}$ with built-in control of power and current, respectively. Both lasers emit collimated, single-mode, Gaussian beams with a full width at half maximum spot size of $\sim 4.5 \mathrm{~mm}$, which were measured using a BeamTrack 10A-PPS thermal sensor (Ophir Photonics). A FLIR SC655 infrared camera was used to record the temperature of samples in all tests. Thermal imaging provides a method to noninvasively monitor the sample temperature during processing. The camera has an array of $640 \times 480$ pixels and a maximum frame rate of $200 \mathrm{fps}$. This camera is sensitive from 7.5 to $14 \mu \mathrm{m}$, which is ideal for sensing temperature in the ranges that we are studying $\left(35^{\circ} \mathrm{C}\right.$ to $\left.77^{\circ} \mathrm{C}\right)$. All studies

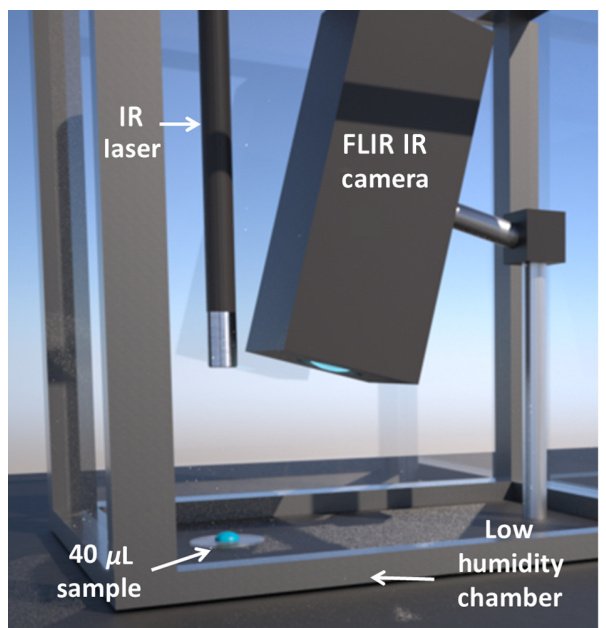

Fig. 1 Experimental setup of LAD technique within a controlled low $\mathrm{RH}$ chamber.

were performed in a humidity-controlled environment that was kept at $\sim 11 \%$ relative humidity $(\mathrm{RH})$. This was achieved by pumping dried air into a chamber containing the experimental setup and monitoring the RH with a temperature and $\mathrm{RH}$ logger (ONSET UX100-011). Maintaining a low RH expedited the drying process. ${ }^{21}$

All samples in the studies consisted of $40-\mu \mathrm{L}$ droplets containing a model protein, egg white lysozyme (Worthington Biochemical LS002933), dissolved in drying solution (DS) at a concentration of $0.5 \mathrm{mg} / \mathrm{mL}$. This was verified using the absorption of light at $280 \mathrm{~nm}$ with a microplate spectrophotometer (Bio-Tek Synergy HT). The DS consisted of $0.2 \mathrm{M}$ disaccharide trehalose in $0.33 \times$ phosphate-buffered solution. ${ }^{22}$ The dry weight of the DS was determined through bake-out method to be $7.01 \%$ the mass of a sample. Dry weight was adjusted to include the mass of the protein based on its concentration to determine the total dry weight.

For each test, a $40-\mu \mathrm{L}$ droplet of the protein/DS was deposited onto a substrate and the initial mass was determined gravimetrically using a balance (RADWAG AS 82/220.R2) accurate to $0.01 \mathrm{mg}$. The sample was then moved into the humidity chamber for laser irradiation at a specific processing power that was held constant. The maximum temperature of the sample was monitored during processing using the thermal camera. This temperature was referred to as the processing temperature $\left(T_{\max }\right)$ and recorded as a function of time for each sample. It is important to note that the ambient temperature of the chamber $\left(\sim 23^{\circ} \mathrm{C}\right)$ was kept constant during processing. After irradiation, the sample was removed from the humidity chamber and immediately massed again. EMC, which is a measure of the amount of water relative to the dry mass of a sample, was calculated as

$\mathrm{EMC}=\frac{m_{\mathrm{f}}-m_{\mathrm{s}}-m_{\mathrm{dw}}}{m_{\mathrm{dw}}}$

where $m_{\mathrm{f}}$ is the mass of the final sample, including the mass of the substrate, $m_{\mathrm{s}}$, and $m_{\mathrm{dw}}$ is the calculated dry weight of the initial sample.

Several different sets of processing parameters were tested and EMC as a function of LAD processing time ( 0 to $60 \mathrm{~min})$ 
Table 1 Processing parameters tested for LAD drying. Wavelength, processing power, and substrate were varied.

\begin{tabular}{|c|c|c|c|c|c|}
\hline $\begin{array}{l}\text { Wavelength } \\
(\mathrm{nm})\end{array}$ & Substrate & $\begin{array}{c}\text { Beam } \\
\text { size } \\
(\mathrm{mm})\end{array}$ & $\begin{array}{l}\text { Irradiance } \\
\left(\mathrm{W} / \mathrm{cm}^{2}\right)\end{array}$ & $\begin{array}{l}\text { Processing } \\
\text { power(W) }\end{array}$ & $T_{\max }\left({ }^{\circ} \mathrm{C}\right)$ \\
\hline \multirow[t]{6}{*}{1850} & Coverslip & 4.01 & 0.88 & 0.11 & $35.0 \pm 2.4$ \\
\hline & & 4.01 & 1.42 & 0.18 & $42.4 \pm 2.1$ \\
\hline & & 4.01 & 4.04 & 0.51 & $77.6 \pm 1.2$ \\
\hline & Filter paper & 24.05 & 0.18 & 0.86 & $36.4 \pm 0.9$ \\
\hline & & 24.05 & 0.29 & 1.36 & $43.9 \pm 0.7$ \\
\hline & & 24.05 & 1.01 & 4.60 & $72.5 \pm 0.3$ \\
\hline \multirow[t]{2}{*}{1064} & Coverslip & 4.86 & 16.15 & 3.00 & $35.2 \pm 0.9$ \\
\hline & & 4.86 & 26.92 & 5.00 & $43.0 \pm 1.8$ \\
\hline
\end{tabular}

was determined (see Table 1). Processing power was chosen for each laser to correspond to a specific processing temperature $\left(T_{\max }\right)$.

Each experiment was repeated three times $(N=3)$, with the exception of the $1064-\mathrm{nm}$ laser at $T_{\max }=43.0^{\circ} \mathrm{C} \pm 1.8^{\circ} \mathrm{C}$ on coverslips at 30 and $60 \mathrm{~min}$ of processing $(N=20)$ to test EMC repeatability, respectively. In addition, identical samples were allowed to air dry in the RH chamber as a control.

For the 1850-nm laser source, three laser processing powers were tested with each resulting in a different processing temperature of the sample during processing. We chose to test various laser processing powers to determine the effect of processing temperature on drying rate. Two different substrates were tested using the 1850-nm laser: 18-mm-diameter borosilicate glass coverslips (Fisherbrand 12-546) and 8-mm-diameter borosilicate glass microfiber filter paper (Whatman 1821-021). The glass coverslips allow for easy recovery and rehydration of the proteins, while the filter paper is used in diagnostic assays. On the glass coverslips, the samples were droplets roughly $2 \mathrm{~mm}$ in thickness with a diameter of roughly $7 \mathrm{~mm}$. A $1.0 \mathrm{ND}$ filter (Newport 5214-A) was placed in line with the 1850-nm laser for processing samples on coverslips since the lowest available power setting caused excessive heating. On the filter paper, the samples dispersed in the paper such that they were a constant thickness of $0.675 \mathrm{~mm}$, with diameter $8 \mathrm{~mm}$. Filter paper samples have a constant sample thickness in comparison to their droplet counterparts. The Gaussian beam of the 1850-nm laser was flattened using a reflective $6 \times$ beam expander (Thorlabs BE06R) to allow for even heating across these samples. Samples were placed in the middle of the expanded beam where there was minimal change in beam profile.

With the 1064-nm laser, two different laser processing powers were used for processing. Due to the lower absorption coefficient of water at $1064 \mathrm{~nm}$, we were not able to achieve sample temperatures above about $44^{\circ} \mathrm{C}$ even at the maximum power output of the laser. In addition, only glass coverslips were used as the substrate for these tests. Beam shaping the $1064 \mathrm{~nm}$ did not give a high enough energy density to achieve necessary processing temperatures to process filter paper samples.

\subsection{Protein Functionality}

The effect of LAD processing on protein functionality was tested by processing lysozyme in the $40-\mu \mathrm{L}$ droplets on glass coverslips for 30 and $60 \mathrm{~min}(N=3)$ using the 1064-nm laser at $5.0 \mathrm{~W}\left(T_{\max } \sim 44^{\circ} \mathrm{C}\right)$. The functionality of lysozyme in each sample was then measured using the assay described by Worthington Biochemical for the rate of lysis of Micrococcus lysodeikticus cells. ${ }^{23}$ The assay was altered to fit a 96-well format and measured the decrease in turbidity as a function of time at $450 \mathrm{~nm}, \Delta A_{450} / \mathrm{min}$, referred to as the response rate. We calculated the specific activity of each sample using the measured concentration and $\Delta A_{450} / \mathrm{min}$. Before assaying, samples were rehydrated and then diluted with deionized water to a concentration of $0.1 \mathrm{mg} / \mathrm{mL}$ of lysozyme to achieve a linear response rate for the assay. The concentration of lysozyme for each sample was verified by measuring the absorption at $280 \mathrm{~nm}$.

A $500-\mu \mathrm{L}$ volume of the sample was also heated in a water bath at $\sim 44^{\circ} \mathrm{C}$ for 30 and 60 min to compare the effect of heating without drying. Three 40- $\mu$ l droplets were taken from this sample and diluted with deionized water to a concentration of $0.1 \mathrm{mg} / \mathrm{mL}$ of lysozyme for use in the functionality assay. It should be noted that the temperature of the LAD samples decreased during processing due to evaporative cooling, while the water bath samples were held at a near constant temperature.

\section{Results and Analysis}

\subsection{End Moisture Contents Curves and Thermal Histories}

Drying curves summarizing the results are shown in Fig. 2. Drying curves are graphs of EMC versus processing time and allow easy comparison of the EMC resulting from different sets of processing parameters. Table 2 summarizes the resulting EMC, sample temperatures, and predicted glass transition temperatures for 60 -min processing times for both lasers and for air drying.

Air drying resulted in much slower drying and much larger EMC's at all processing times than either laser source. During air drying, evaporation reduces the temperature of the sample

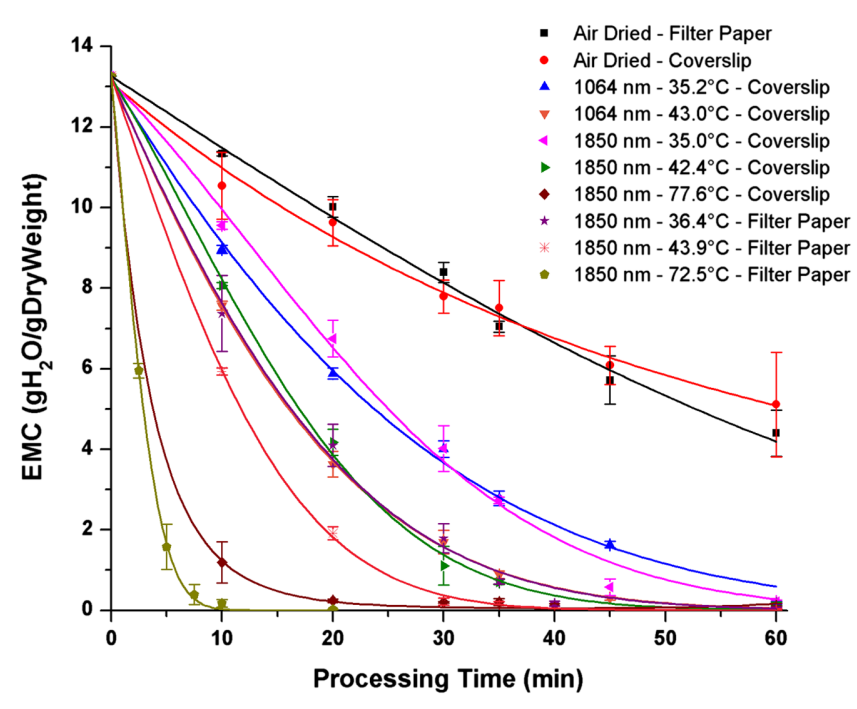

Fig. 2 EMC as a function of processing time for various processing parameters. 
Table 2 EMC for each set of processing parameters and their associated glass transition temperatures.

\begin{tabular}{|c|c|c|c|c|c|}
\hline Wavelength $(\mathrm{nm})$ & Substrate & $T_{\max }\left({ }^{\circ} \mathrm{C}\right)$ & $T_{\mathrm{p}}(\min )$ & EMC $\left(\mathrm{gH}_{2} \mathrm{O} / \mathrm{gDryWeight}\right)$ & $T_{\mathrm{g}}\left({ }^{\circ} \mathrm{C}\right)$ \\
\hline \multirow[t]{6}{*}{1850} & Coverslip & $35.0 \pm 2.4$ & 60 & $0.22 \pm 0.04$ & -25.4 \\
\hline & & $42.4 \pm 2.1$ & 60 & $0.16 \pm 0.04$ & -6.7 \\
\hline & & $77.6 \pm 1.2$ & 60 & $0.14 \pm 0.03$ & 1.0 \\
\hline & Filter paper & $36.4 \pm 0.9$ & 60 & $0.06 \pm 0.03$ & 44.1 \\
\hline & & $43.9 \pm 0.7$ & 60 & $0.03 \pm 0.02$ & 68.3 \\
\hline & & $72.5 \pm 0.3$ & 20 & $0.03 \pm 0.01$ & 68.3 \\
\hline \multirow[t]{2}{*}{1064} & Coverslip & $43.0 \pm 1.8$ & 60 & $0.17 \pm 0.04$ & -10.3 \\
\hline & & $35.2 \pm 0.9$ & 60 & $0.19 \pm 0.03$ & -16.8 \\
\hline \multirow[t]{2}{*}{ Air drying } & Coverslip & $\mathrm{N} / \mathrm{A}$ & 60 & $5.11 \pm 1.3$ & -126.5 \\
\hline & Filter paper & $\mathrm{N} / \mathrm{A}$ & 60 & $4.40 \pm 0.6$ & -125.2 \\
\hline
\end{tabular}

and this decreases the evaporation rate. With LAD, the laser adds energy to the drop to counteract and slow the effects of evaporative cooling.

We tested two laser wavelengths in this study, 1064 and $1850 \mathrm{~nm}$, and compared the drying capabilities of these two wavelengths for drops deposited on the glass coverslip substrate. To compare the resulting drying curves for these two wavelengths, we compared laser powers that resulted in the same processing temperatures. The temperatures were measured using the thermal camera. We compare the performance of the lasers at processing temperatures of $35^{\circ} \mathrm{C}$ and $42^{\circ} \mathrm{C}$. For both lasers, as the processing temperature increased from $35{ }^{\circ} \mathrm{C}$ to $42{ }^{\circ} \mathrm{C}$, the resulting EMC after $60 \mathrm{~min}$ is lower, as expected. The EMCs at $60 \mathrm{~min}$ for both laser sources were approximately the same. The power density used with the 1850 -nm laser was much lower than that of the 1064-nm laser. This is because the water absorption coefficient for the $1850-\mathrm{nm}$ laser is much higher than the 1064-nm laser. Therefore, even with less power per unit area, the sample heated at a similar rate due to more of that power being absorbed by the water. We then tested an additional temperature, $77^{\circ} \mathrm{C}$ with the 1850-nm laser and saw an even further decrease of EMC. Overall, we see that both lasers have similar drying curves at the same processing temperature. The 1850-nm laser yields the lowest EMC but at the cost of a higher processing temperature. The low power needed to achieve this drying is an advantage but could also mean uneven sample heating because of the short penetration depth.

Looking at the drying curves in detail (see Fig. 3) reveals an interesting result when comparing drying curves for each wavelength at similar processing temperatures. We would expect that processing the sample at the same temperature regardless of wavelength would generate the same drying curve; instead we see that wavelength does affect the drying rate. For short processing times $(<30 \mathrm{~min})$, the 1064-nm laser provides lower EMCs, but for processing times $>30 \mathrm{~min}$, the 1850 -nm laser results in lower EMC until the drying curves converge at $60 \mathrm{~min}$. This happens at both low and intermediate laser powers $\left(35^{\circ} \mathrm{C}\right.$ and $42{ }^{\circ} \mathrm{C}$ sample temperatures). Also, the slope of the drying curve, which indicates the drying rate, is initially steeper

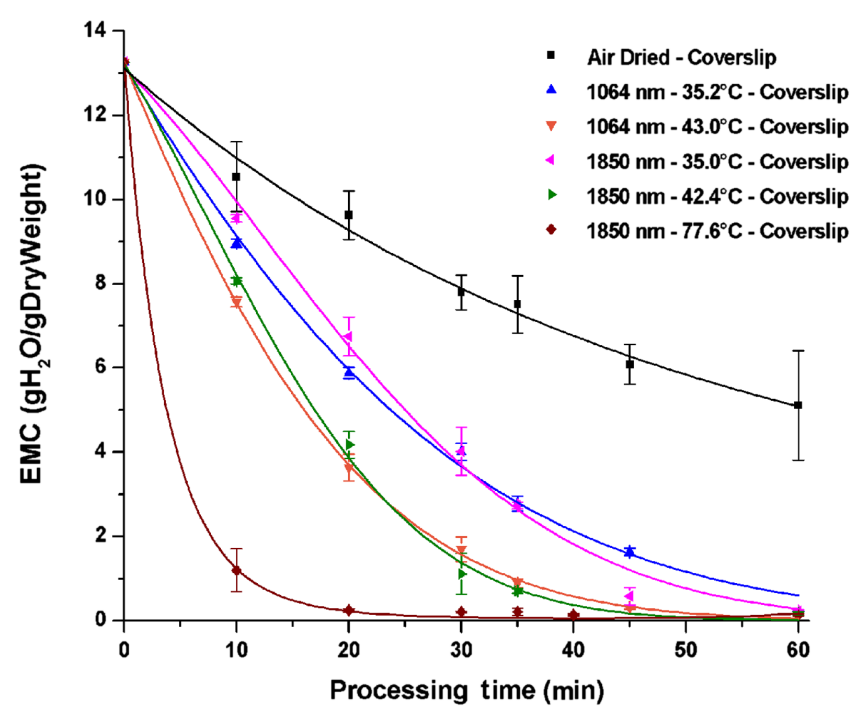

Fig. $3 \mathrm{EMC}$ as a function of processing time for drying on coverslips.

for the 1064-nm laser, but after $30 \mathrm{~min}$ of processing time, the drying rate of the $1850-\mathrm{nm}$ laser is steeper. The absorption coefficient for the 1850-nm laser source may account for these differences in the drying curves. Initially, 1064-nm light couples effectively because there is more water to interact with. As the water evaporates, the 1064-nm source couples less strongly causing the amount of energy deposited to decrease, and subsequently the slope of the drying curve flattens. A possible method to combat this issue is to increase the power of the 1064-nm source during processing.

Recall that a larger sample size, $N=20$ for the 1064-nm laser at $\sim 43.0^{\circ} \mathrm{C}$ at processing times of 30 and 60 min was used to test repeatability. The average EMC and standard deviation at $30 \mathrm{~min}$ were $1.69 \pm 0.29 \mathrm{gH}_{2} \mathrm{O} / \mathrm{gDryWeight}$ and at $60 \mathrm{~min}$ were $0.15 \pm 0.03 \mathrm{gH}_{2} \mathrm{O} / \mathrm{gDryW}$ eight. The decrease in standard deviation implies that the drier the sample gets the more repeatable the EMC, likely because of the precise energy deposition of LAD. High repeatability of EMC is very important for achieving a small spread in storage temperatures. 


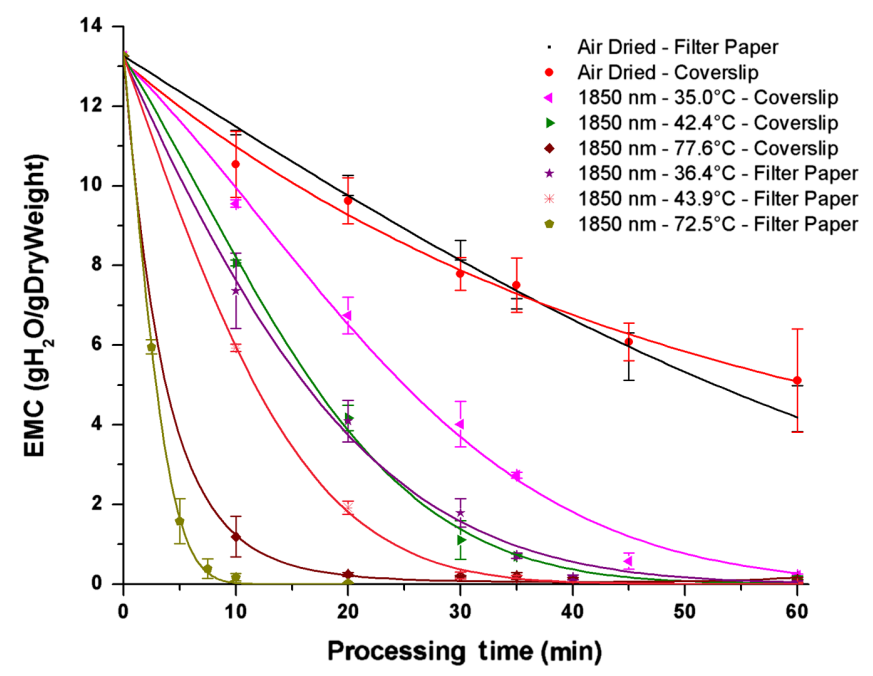

Fig. 4 EMC as a function of processing time for drying with 1850-nm laser.

We tested two substrates-glass coverslips and filter paperwith the 1850-nm laser (see Fig. 4 and Table 2) at sample temperatures of $35^{\circ} \mathrm{C}$ and $42^{\circ} \mathrm{C}$. Drying on the glass coverslips was slower than drying on the filter paper (for comparable sample temperatures); filter paper gave the lowest EMC. This is likely

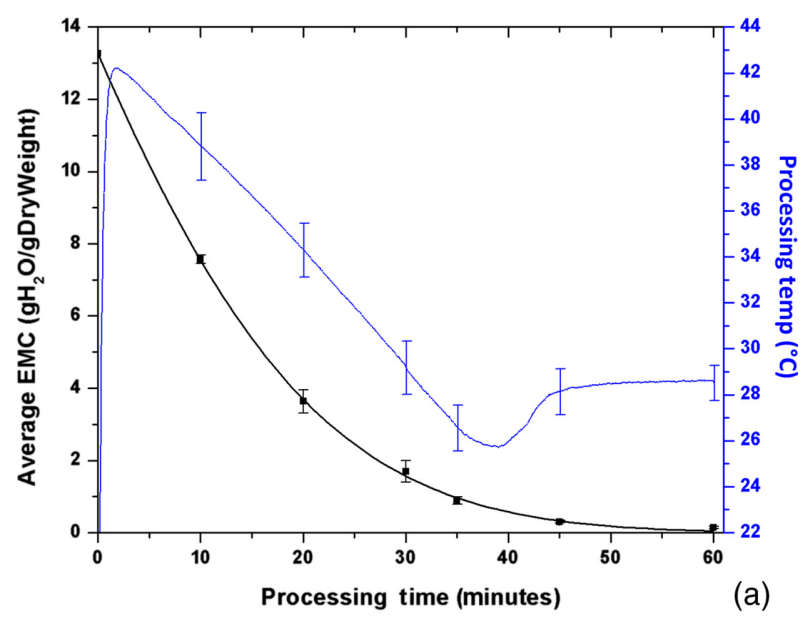

the result of the difference in surface tension of the sample on coverslips versus filter paper. On the coverslips, the sample maintains a hemispherical droplet shape, while on the filter paper the drop spreads out and assumes a two-dimensional planar structure. The surface tension of the droplets on coverslips decreases the evaporation rate. The filter paper breaks up the droplet and spreads out so it is thinner, increasing surface area and reducing the surface tension. We tested the 1850-nm laser source of the filter paper at a higher power that resulted in a processing temperature of $72^{\circ} \mathrm{C}$. At the high temperature, the EMC at $20 \mathrm{~min}$ was comparable to $60 \mathrm{~min}$ of processing at a temperature of $36^{\circ} \mathrm{C}$ on filter paper.

All light-assisted processing provided EMCs with good repeatability as measured by the small standard deviation of the mean at all processing times and for all laser parameters (see Table 2). This was not the case for air drying which had a very high variability in EMC. The repeatability of LAD is likely due to precise and repeatable energy deposition into the samples during processing.

The processing temperature was recorded during processing for each sample to give thermal history. The average processing temperature for the 60-min samples was averaged for an $N=3$ thermal history. In Fig. 5, we compare the EMC to the corresponding thermal history for each set of processing parameters at similar processing temperatures. In all cases, there is initial heating of the drop as the laser energy is added. Once

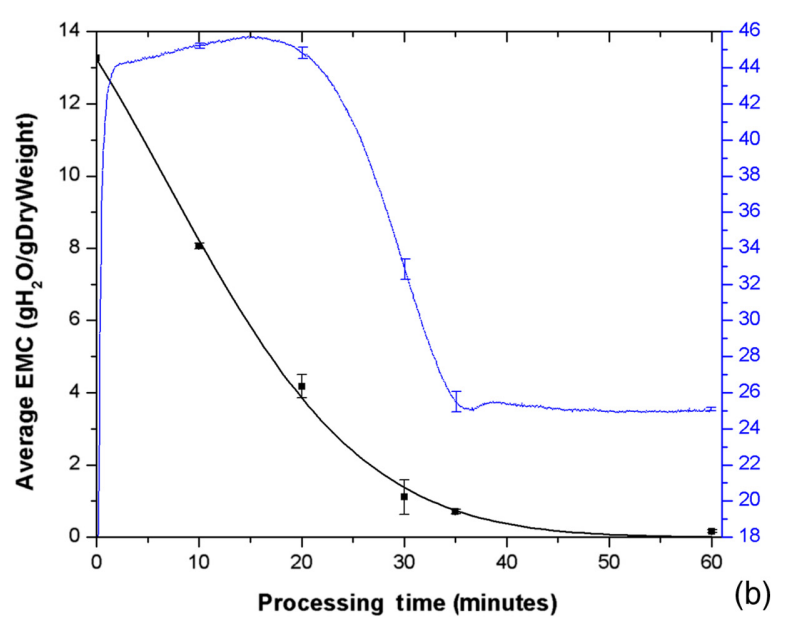

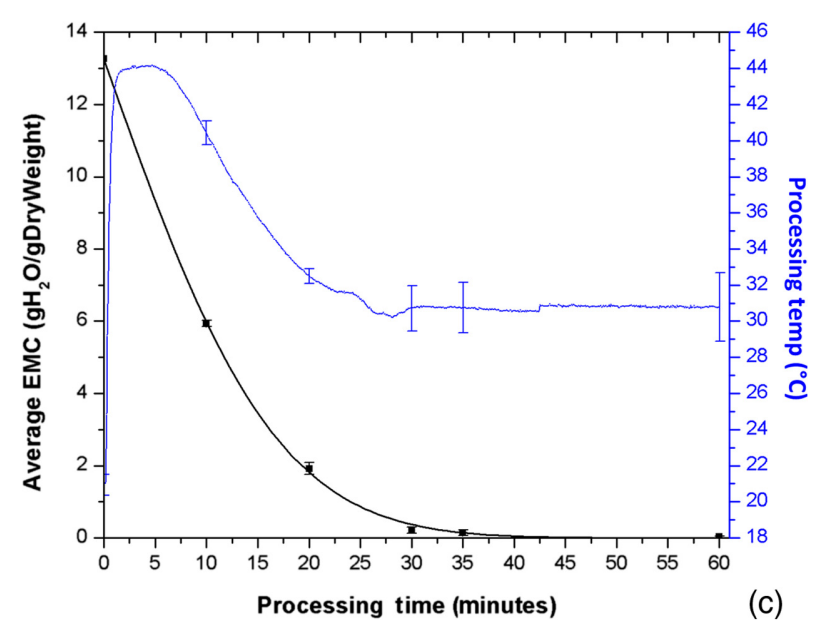

Fig. 5 Thermal histories with EMC curves for LAD processing of (a) $1064 \mathrm{~nm}$ on coverslips, (b) $1850 \mathrm{~nm}$ on coverslips, and (c) $1850 \mathrm{~nm}$ processing on filter paper for similar processing temperatures. 


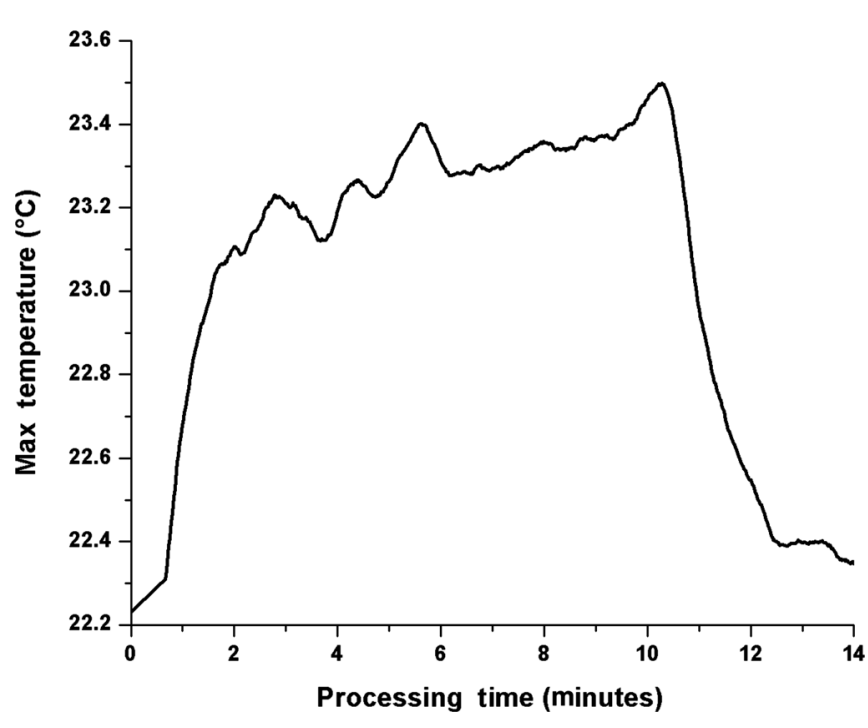

Fig. 6 Thermal history of borosilicate glass coverslip without sample under illumination with 1064-nm laser at $5 \mathrm{~W}$. This is the same processing parameters associated with Fig. 5(a). This heating curve corresponds to the heating observed during the end of LAD processing after most of the water has been removed from the sample.

evaporation begins, cooling occurs. All samples experience this cooling effect during processing but at different times. Evaporative cooling is removing more energy than the laser adds through absorption. As the sample evaporates, the laser couples less strongly. To overcome evaporative cooling, we would need to increase laser power during processing.

The processing temperature for the $1064 \mathrm{~nm}$ on coverslips did not maintain peak temperature throughout LAD, evaporative cooling was the dominate mechanism, and the sample cooled until $\sim 40 \mathrm{~min}$ of processing. Heating resumed again and plateaued at $28^{\circ} \mathrm{C}$, this was most likely because of the heating of the coverslip. Figure 6 shows the heating of a glass coverslip with the 1064-nm laser at $5 \mathrm{~W}$. We see the same characteristic plateau that is observed at the end of the thermal histories of the samples.

Processing temperature for the 1850-nm laser on coverslips showed an increase in sample temperature for the first $20 \mathrm{~min}$ because it coupled strongly with water in the sample and delivered enough energy to overcome evaporative cooling. At $\sim 20 \mathrm{~nm}$, the volume of water had decreased; therefore, less energy was being delivered and evaporation caused a drop in temeprature. After $35 \mathrm{~min}$ of processing, we saw a small increase in temperature followed by a plateau at $25^{\circ} \mathrm{C}$ caused by the heating of the coverslip. Finally, processing temperature for the 1850-nm laser on filter paper showed the same increase in sample temperature followed by a decrease from evaporative cooling and a small increase from substrate heating that was seen in the thermal history of 1850-nm coverslip drying but on a shorter timescale. Also note that the substrate heating of the filter paper plateaus at a higher temperature than the coverslip, this is likely because the filter paper fibers scatter the incoming light increasing the optical path length of the light in the substrate and allowing for more absorption. For all LAD drying, the point at which substrate heating took effect was at an $\mathrm{EMC}=0.58 \pm 0.04 \mathrm{gH}_{2} \mathrm{O} / \mathrm{gDryWeight}$. This was because the volume of water left in the sample was low enough that there was negligible water absorption and laser energy was absorbed by the substrate.

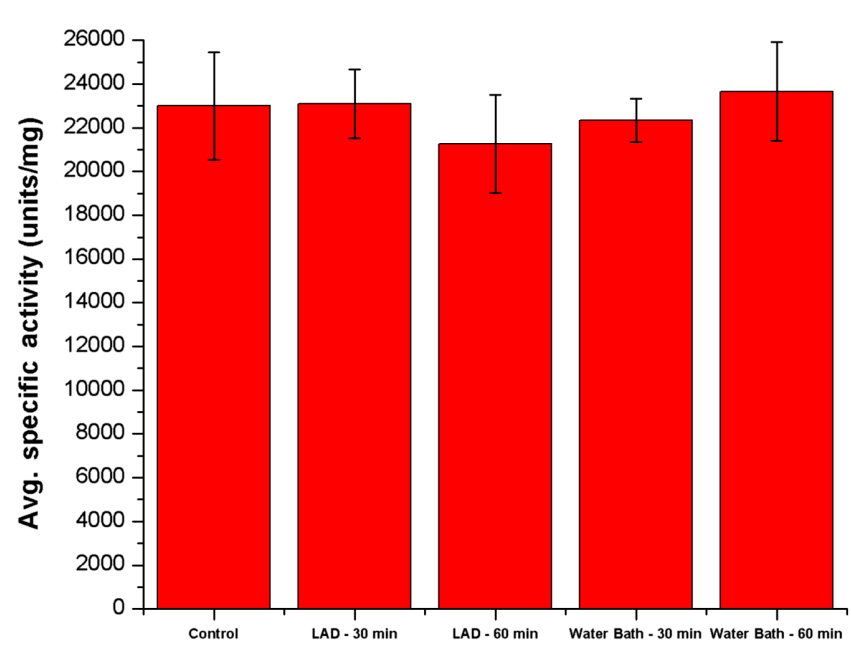

Fig. 7 Average specific activity of lysozyme for refrigerated control solution, LAD processed solution, and solution incubated at LAD processing temperature $\left(\sim 44^{\circ} \mathrm{C}\right)$.

\subsection{Protein Functionality}

Figure 7 shows the average specific activity of control samples, 30 and 60 min LAD processed samples, and samples incubated at a constant temperature comparable to the LAD processing temperature of $\sim 44^{\circ} \mathrm{C}$. Specific activity is a measure of the ability of a protein to perform its function. In this assay, it is dependent on the rate of lysis of Micrococcus lysodeikticus by lysozyme. A high specific activity indicates the proteins are undamaged and functional. We see that all samples have a specific activity $>20,000$ units $/ \mathrm{mg}$, where units are defined according to the Worthington biochemical lysozyme assay. This indicates that all samples are still functional even after drying and/or heating. Note that $60 \mathrm{~min}$ of LAD processing does show a drop in specific activity but the average is still within the standard deviation of the control. This suggests that LAD does not adversely affect the functionality of the lysozyme protein during processing. Figure 8 shows average specific activity as a function of corresponding EMC of the same set of samples. We see no correlation between loss of moisture and functionality

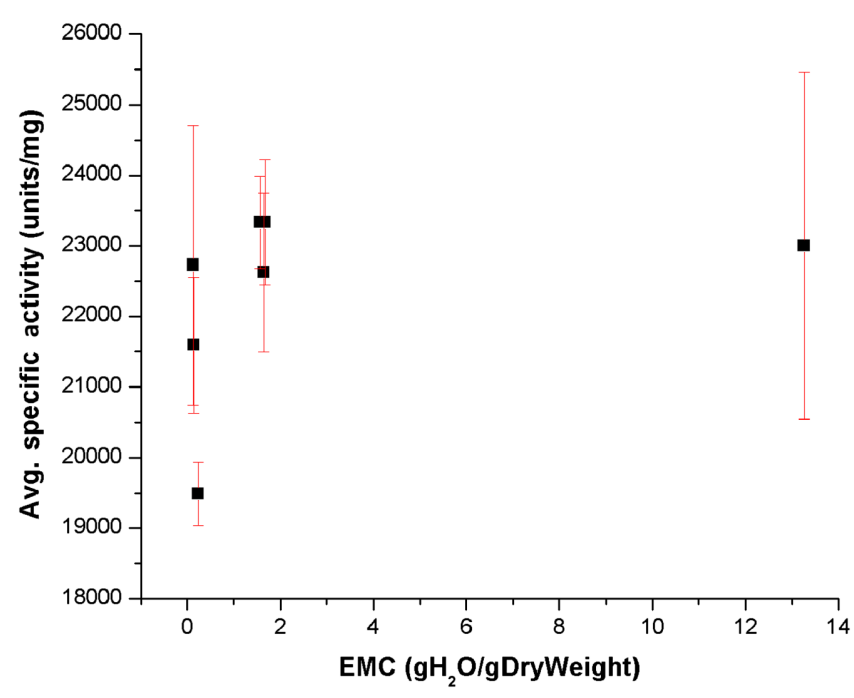

Fig. 8 Average specific activity of lysozyme as a function of EMC. 
of the protein lysozyme. These initial findings imply that processing to these low EMCs will not reduce activity.

\section{Discussion}

Both the 1064- and 1850-nm laser sources show promise for use in the preparation of trehalose glasses to be used for the preservation of proteins or other biologics. These wavelengths have different absorption in water and so deliver energy differently into the sample. This means that you can tune LAD to fit the needs of the sample being processed by varying the wavelength. Table 2 shows the predicted storage temperatures $\left(T_{\mathrm{g}}\right)$ for the samples prepared in this study. The estimated $T_{\mathrm{g}}$ for drops dried on coverslips is close to $0^{\circ} \mathrm{C}$. This is an improvement compared to the sub-zero storage temperatures required for some proteins, but on par with lyophilized proteins that require cold chain storage. Finally, the samples did not reach sufficiently low EMC that would have a $T_{\mathrm{g}}$ near room temperature. There are multiple ways to improve this result. Longer processing time is one option; however, with the drying curves flattening out substantially at processing times of $60 \mathrm{~min}$, the additional drying seen past this point would likely be small. Adding more power would increase the sample temperature and would, therefore, increase the drying. With this solution comes the risk of damaging the embedded protein. Last, we could add a surfactant to the solution to decrease surface tension, which would increase the drying rate.

The standard deviation of the technique is low compared to air drying. We found that LAD repeatability increased at lower EMC. This is beneficial because smaller variation in EMC means a smaller range of storage temperatures. It should be noted that gravimetrically determining EMC is only an indicator of the average EMC of the entire sample. This does not show the varying moisture contents across the sample, which would lead to a higher storage temperature that corresponds to the area of highest EMC.

The combination of the 1850-nm source and the filter paper substrate provided the best results. The predicted storage temperatures for the samples processed on filter paper are promising for storage at ambient temperatures. For example, after $60 \mathrm{~min}$ of processing, the 1850-nm laser produced trehalose glasses with potential storage temperatures ranging from $47^{\circ} \mathrm{C}$ to $69^{\circ} \mathrm{C}$.

LAD has the potential to provide a processing and storage method for protein-based drugs and diagnostic assays. Freeze drying can take multiple hours to complete. LAD processing times of an hour are considerable improvements. LAD offers a relatively inexpensive, compact method for initial processing, and anhydrous preservation allows for low-maintenance storage after samples have been packaged.

Protein functionality testing demonstrates the effectiveness of LAD for processing the protein lysozyme without altering its functionality. Initial results indicate that low EMC will not lead to denaturation. While the elevated processing temperature in this study did not lead to denaturation, further studies will test the functionality of proteins before and after processing at higher temperatures that are known to cause thermal denaturation. Ideally, we would be able to process samples at very high temperatures for fast drying while maintaining protein functionality. These tests will give us the temperature threshold for processing.

In future studies, we will use Raman spectroscopy to determine the sugar content within the dried samples and, thus, the uniformity of the drying process for samples dried on glass coverslips. Abazari et al. ${ }^{24}$ have successfully used Raman spectroscopy to quantify the residual water contents of spin-dried cells. Polarized light microscopy will be used to measure the homogeneity of amorphous materials and locate areas of crystallization. Localized areas of crystallization in the samples can act as nucleation points and increase the rate of crystallization as well as lead to physical stress on biologics embedded in the matrix. ${ }^{25}$ Polarized light microscopy has been used previously to analyze the presence of crystallization in sugars. ${ }^{26}$ White light interferometry will be used to measure sample thickness and profile. Previous studies by Chakraborty et al. ${ }^{22}$ revealed significant differences in the dried drop morphology of air-dried versus microwaved processed drops. Samples that were processed using microwave-assisted drying had much more uniform distributions of the dried trehalose matrix than the air-dried samples.

\section{Disclosures}

The authors have no relevant financial interests in the paper and no other potential conflicts of interest to disclose.

\section{Acknowledgments}

This work was supported, in part, by funds provided by The University of North Carolina (UNC) at Charlotte Faculty Research Grants Program, by the Charlotte Research Institute Commercial Development Grant Program, and the Center for Biomedical Engineering and Science at UNC Charlotte.

\section{References}

1. B. Leader, Q. J. Baca, and D. E. Golan, "Protein therapeutics: a summary and pharmacological classification," Nat. Rev. Drug Discov. 7(1), 21-39 (2008).

2. P. J. Mease et al., "Etanercept in the treatment of psoriatic arthritis and psoriasis: a randomised trial," Lancet (London, England) 356(9227), 385-390 (2000).

3. V. Romanov et al., "A critical comparison of protein microarray fabrication technologies," Analyst 139(6), 1303-1326 (2014).

4. J. J. Hill, E. Y. Shalaev, and G. Zografi, "Thermodynamic and dynamic factors involved in the stability of native protein structure in amorphous solids in relation to levels of hydration," J. Pharm. Sci. 94(8), 16361667 (2005).

5. M. Ehrnsperger et al., "Stabilization of proteins and peptides in diagnostic immunological assays by the molecular chaperone Hsp25," Anal. Biochem. 259(2), 218-225 (1998).

6. J. Bjerketorp et al., "Advances in preservation methods: keeping biosensor microorganisms alive and active," Curr. Opin. Biotechnol. 17(1), 43-49 (2006).

7. G. Adams, "Lyophilization of vaccines: current trends," Methods Mol. Med. 87, 223-244 (2003).

8. L. L. Chang et al., "Mechanism of protein stabilization by sugars during freeze-drying and storage: native structure preservation, specific interaction, and/or immobilization in a glassy matrix?" J. Pharm. Sci. 94(7), 1427-1444 (2005).

9. Pierce Biotechnology, Inc., "Protein stability and storage," TR0043.1, Pierce Biotechnology, Inc., Rockford, Illinois (2005).

10. G. D. Elliott, N. Chakraborty, and D. Biswas, "Anhydrous preservation of mammalian cells: cumulative osmotic stress analysis," Biopreserv. Biobank. 6(4), 253-260 (2008).

11. L. Yu, "Amorphous pharmaceutical solids: preparation, characterization and stabilization," Adv. Drug Deliv. Rev. 48(1), 27-42 (2001).

12. J. H. Crowe and L. M. Crowe, "Preservation of mammalian cells-learning nature's tricks," Nat. Biotechnol. 18(2), 145-146 (2000).

13. S. D. Allison et al., "Hydrogen bonding between sugar and protein is responsible for inhibition of dehydration-induced protein unfolding," Arch. Biochem. Biophys. 365(2), 289-298 (1999). 
14. D. Champion, M. Le Meste, and D. Simatos, "Towards an improved understanding of glass transition and relaxations in foods: molecular mobility in the glass transition range," Trends Food Sci. Technol. 11(2), 41-55 (2000).

15. M. Gordon and J. S. Taylor, "Ideal copolymers and the second-order transitions of synthetic rubbers. I. Non-crystalline copolymers," J. Appl. Chem. 2(9), 493-500 (2007).

16. T. Chen, A. Fowler, and M. Toner, "Literature review: supplemented phase diagram of the trehalose-water binary mixture," Cryobiology 40(3), 277-282 (2000).

17. N. Chakraborty et al., "Trehalose transporter from African Chironomid larvae improves desiccation tolerance of Chinese hamster ovary cells," Cryobiology 64(2), 91-96 (2012).

18. H. Y. Elmoazzen et al., "Further optimization of mouse spermatozoa evaporative drying techniques," Cryobiology 59(1), 113-115 (2009).

19. S. L. Cellemme et al., "Advancing microwave technology for dehydration processing of biologics," Biopreserv. Biobank. 11(5), 278-284 (2013).

20. A. Vogel and V. Venugopalan, "Mechanisms of pulsed laser ablation of biological tissues," Chem. Rev. 103(2), 577-644 (2003).
21. M. A. Young et al., "Light assisted drying (LAD) for protein stabilization: optimization of laser processing parameters," Proc. SPIE 10081, 100810R (2017).

22. N. Chakraborty et al., "A role for microwave processing in the dry preservation of mammalian cells," Biotechnol. Bioeng. 100(4), 782-796 (2008).

23. K. Worthington and V. Worthington, "Worthington Enzyme Manual," Worthington Biochemical Corporation, Lakewood, New Jersey (2011).

24. A. Abazari et al., "A Raman microspectroscopy study of water and trehalose in spin-dried cells," Biophys. J. 107(10), 2253-2262 (2014).

25. A. Saleki-Gerhardt, "Non-isothermal and isothermal crystallization of sucrose from the amorphous state," Pharm. Res. 11(8), 1166-1173 (1994).

26. M. F. Mazzobre, J. M. Aguilera, and M. P. Buera, "Microscopy and calorimetry as complementary techniques to analyze sugar crystallization from amorphous systems," Carbohydr. Res. 338(6), 541-548 (2003).

Biographies for the authors are not available. 